\title{
Nonlinear heat-transfer macromodeling for MEMS thermal devices
}

\author{
Yao-Joe Yang and Kuo-Yeh Shen \\ Department of Mechanical Engineering, National Taiwan University, No. 1 Roosevelt Rd., \\ Sec. 4, Taipei, Taiwan, Republic of China \\ E-mail: yjy@ntu.edu.tw
}

Received 20 August 2004, in final form 26 October 2004

Published 8 December 2004

Online at stacks.iop.org/JMM/15/408

\begin{abstract}
In this paper, we present a nonlinear heat-transfer macromodeling technique using the trajectory piecewise-linear model-order-reduction (TPWLMOR) method. A 3D nonlinear heat-transfer model, which is capable of accounting for the temperature-dependent material properties as well as the radiation effect, is implemented using the finite-difference method (FDM). The numerical models generated by the FDM are reduced into compact models using the TPWLMOR technique, which is based on the concept of a piecewise-linear approximation and an Arnoldi-based model-orderreduction (MOR) algorithm. Nonlinear macromodeling case studies of different MEMS thermal devices are demonstrated using the TPWLMOR technique. The calculated steady and transient characteristics of the thermal devices are discussed. In terms of computational cost, the TPWLMOR models are at least 2 orders of magnitude faster than the original nonlinear full-meshed models with a negligible compromise in accuracy. The simulated results by the TPWLMOR models are also verified with the experimentally measured results.
\end{abstract}

(Some figures in this article are in colour only in the electronic version)

\section{Introduction}

There are many MEMS devices employing thermal actuating or sensing mechanisms [1-4]. In order to perform system-level dynamical analyses or closed-loop control for those devices, compact and accurate thermal models are required [5]. The lumped-element method is the most straightforward approach to create thermal compact models $[5,6]$. When using the lumped-element approach, the device has to be partitioned into a few smaller parts which have simple geometrical shapes. For each partitioned part, the heat capacity is approximated as a lumped heat capacity $\left(C_{\mathrm{th}}\right)$, and the energy transportation mechanisms, such as conduction, convection and radiation effects, are approximated as lumped heat resistors $\left(R_{\mathrm{th}}\right)$. By appropriately assembling the lumped elements of each part, the overall heat-transfer model of the original system can be built. The lumped-element approach is quite efficient for the devices with simple geometries, but its accuracy decreases significantly as the geometries of devices are very complex.

Therefore, the works published in [7] and [8] propose the methodologies of generating linear heat-transfer reduced- order models (macromodels) from complex three-dimensional (3D) FDM/FEM solid models based on an Arnoldi-based model-order-reduction (MOR) technique [9, 10]. Since the macromodels are reduced from the original 3D solid model of the system, potentially they are not only as efficient as the reduced models built by the lumped-element approach, but are also capable of capturing the geometric characteristics which the lumped-element approach lacks. However, these methodologies are not capable of accounting for the nonlinear effects due to material properties as well as radiation. Therefore, the compact models generated by these methodologies become inaccurate as nonlinear effects are noticeable.

For nonlinear macromodeling, the Karhunen-Loeve/ Galerkin approaches $[11,12]$ are based on the basis functions extracted from an ensemble of snapshots of the physical fields (e.g., pressure distribution or temperature distribution) under prescribed actuation conditions, and have been proved to be very efficient and accurate for modeling nonlinear systems. However, it requires very expensive training FEM/FDM runs to provide enough snapshot data for extracting basis functions. 
Nonlinear heat-transfer macromodeling for MEMS thermal devices

In this work, we present an approach to create nonlinear heat-transfer macromodels from 3D FDM/FEM solid models using the trajectory piecewise-linear model-order-reduction (TPWLMOR) technique $[13,14]$. The TPWLMOR technique is based on an Arnoldi-based model-order-reduction (MOR) algorithm and the concept of piecewise-linear approximation. The MOR technique is used to find the linearization points and to reduce full-meshed models into linearized compact models. The piecewise-linear approximation, on the other hand, is used to build the nonlinear compact models which mimic the behavior of the original nonlinear system based on the weighted combination of the linearized full-meshed models at different linearization points. We also consider the air conduction effect and the conduction shape factor to account for the heat conduction from suspended structures to the substrate. Verification with experimentally measured results for different nonlinear thermal devices will also be presented and discussed.

This paper is organized as follows. The governing equations for nonlinear heat-transfer effect are described in section 2. The algorithm of TPWLMOR is presented in section 3. In section 4, two simulated cases of nonlinear thermal MEMS devices are presented. The comparisons between the results by the linear full-meshed model, the nonlinear full-meshed model and the TPWLMOR models are demonstrated and discussed. The verifications with experimentally measured results are also provided in this section. Finally, section 5 concludes this paper.

\section{Heat-transfer governing equations}

The governing equation for transient heat-transfer problems is the so-called heat equation (Fourier's equation) [15], as shown below:

$\frac{1}{\rho C} \nabla \cdot\left(k_{p}(T) \nabla T\right)+\frac{1}{\rho C} g(\vec{r}, t)=\frac{\partial T}{\partial t} \quad$ Region $\boldsymbol{R}, \quad t>0$

where $g(\vec{r}, t)$ is the volume heat generation at position $\vec{r}$ and at time $t$. Table 1 describes all the variables and the material properties used in this section.

If $g(\vec{r}, t)$ is generated by the Joule heating effect, it can be represented as

$$
g(\vec{r}, t)=J^{2} \rho_{r}(T)=J^{2} \rho_{0}\left(1+\beta\left(T-T_{0}\right)\right) .
$$

The boundary and initial conditions of equation (1) can be represented as

$$
k_{i}(T) \frac{\partial T}{\partial n_{i}}=Q_{\text {conv }}+Q_{\text {rad }}+Q_{\text {cond }} \quad \text { Boundary } S_{i}, \quad t \geqslant 0
$$

$$
T(\vec{r}, t)=F(\vec{r}) \quad \text { Region } \boldsymbol{R}, \quad t=0 .
$$

In equation (3), $Q_{\text {conv }}, Q_{\text {rad }}$ and $Q_{\text {cond }}$ are the heat flux into $\boldsymbol{R}$ through $S_{i}$. $Q_{\text {conv }}$ is the flux due to heat convection, $Q_{\text {rad }}$ is the flux due to radiation and $Q_{\text {cond }}$ is the flux due to conduction. These terms can be represented as follows:

$$
\begin{gathered}
Q_{\mathrm{conv}}=h\left(T_{0}-T\right) \\
Q_{\mathrm{rad}}=\varepsilon \sigma\left(T_{0}^{4}-T^{4}\right)
\end{gathered}
$$

Table 1. The variables used in the heat-transfer governing equations.

\begin{tabular}{ll}
\hline$T$ & Temperature vector \\
$g(\vec{r}, t)$ & Heat generation per unit volume \\
$\vec{r}$ & Position vector \\
$\rho$ & Density \\
$c$ & Specific heat \\
$k_{p}(T)$ & Temperature-dependent thermal conductivity \\
$R$ & Computational domain \\
$F(\vec{r})$ & Initial temperature distribution \\
$J$ & Current density \\
$\rho_{r}(T)$ & Temperature-dependent resistivity \\
$\rho_{0}$ & Resistivity at ambient temperature \\
$T_{0}$ & Ambient temperature \\
$\beta$ & Temperature coefficient of resistivity \\
$S_{i}$ & Boundary of computational domain \\
$k_{i}$ & Thermal conductivity on the $i$ th boundary \\
$n_{i}$ & Normal vector on the boundary \\
$h$ & Convection coefficient \\
$Q_{\text {conv }}$ & Heat flux flow into computational domain due to \\
$Q_{\text {rad }}$ & heat convection \\
$Q_{\text {cond }}$ & Heat flux flow into computational domain due to radiation \\
$S$ & Heat flux flow into computational domain due to \\
$S$ & Shape factor \\
$\varepsilon$ & Stephan-Boltzmann constant \\
$R_{\text {thermal }}$ & Emissivity \\
$l$ & Thermal resistance \\
$w$ & Height of the device \\
$l_{\text {air }}$ & Width of the device \\
$k_{\text {air }}(T)$ & Teight of air gap between device and substrate \\
$l_{n}$ & Height of Sira ${ }_{3}$ Ni ${ }_{4}$ layer \\
$k_{n}$ & Thermal conductivity of Si ${ }_{3}$ Ni $i_{4}$ \\
$l_{s}$ & Height of the substrate \\
$k_{s}$ & Thermal conductivity of substrate \\
$A_{y}$ & Area of the surfaces facing the substrate \\
\hline &
\end{tabular}

$$
Q_{\text {cond }}=\frac{S}{R_{\text {thermal }}}\left(T_{0}-T\right) .
$$

Note that heat conduction from the suspended structure to the substrate through the air film between them is also considered [16]. Equation (8) is the corresponding shape factor used in equation (7) for more accurate modeling [17].

$$
S=\frac{l}{w}\left(\frac{2 l_{\mathrm{air}}}{l}+1\right)+1 \text {. }
$$

Also, in equation (7), the effective thermal resistance $R_{\text {thermal }}$, which represents the heat path from suspended structures to the substrate, is written as three thermal resistances connected in series.

$$
R_{\text {thermal }}=\frac{l_{\text {air }}}{k_{\mathrm{air}}(T) A_{y}}+\frac{l_{n}}{k_{n} A_{y}}+\frac{l_{s}}{k_{s} A_{y}} .
$$

The governing equation (equation (1)) can be approximated using either the finite-element method or the finite-difference method [15]. In this work, we develop a 3D transient heat-transfer solver using the finite-difference-based spatial discretization on the governing equation.

\section{Trajectory piecewise-linear model order reduction}

Although the 3D FDM heat-transfer solver is capable of calculating transient behaviors for 3D geometries with various 
boundary and volume conditions, the computational cost will be very expensive if the $3 \mathrm{D}$ thermal computational domain is very complex. Furthermore, the temperaturedependent material properties, such as thermal conductivities and electric resistivities of the structural layers and the air, make the governing equation exhibit nonlinear behavior. Although the model-order-reduction techniques proposed in [7, 8] can effectively generate efficient macromodels, they only guarantee to be accurate for the linear cases (material properties are temperature independent, and the radiation effect is not included), and therefore are not suitable to be applied to the nonlinear cases directly.

In this work, we propose a nonlinear heat-transfer modeling approach using the TPWLMOR technique. Before discussing the complete procedure of simulating nonlinear thermal systems using TPWLMOR models, we start with the concept of approximating a nonlinear system using the trajectory piecewise-linear (TPWL) technique.

\subsection{Introduction of the trajectory piecewise-linear approximation}

After a finite-difference discretization, the nonlinear fullmeshed heat-transfer model (equation (1)) can be described using the state-space approach:

$$
\frac{\mathrm{d} T(t)}{\mathrm{d} t}=f(T(t))+B(T(t)) u(t)
$$

where $T(t) \in R^{N}$ is the vector of states (temperature distribution) at time $t, N$ is the number of state variables, $f: R^{N} \rightarrow R^{N}$ is a nonlinear vector-valued function, $B(T(t))$ is an input matrix and $u(t)$ is an input signal.

The nonlinear function $f(\cdot)$ can be approximated using Taylor's expansion about a certain state $T_{i}$ (i.e., the $i$ th linearization state).

$$
f(T) \approx f\left(T_{i}\right)+A_{i}\left(T-T_{i}\right)
$$

where $A_{i}$ is the Jacobian of $f(T)$ evaluated at $T=T_{i}$.

$B(\cdot)$ is simplified as

$$
B(T) \approx B\left(T_{i}\right)
$$

After the linearization at the linearization point $T_{i}$, the nonlinear full-meshed system (equation (10)) can be written as a linear full-meshed model:

$$
\frac{\mathrm{d} T(t)}{\mathrm{d} t}=f\left(T_{i}\right)+A_{i}\left(T-T_{i}\right)+B\left(T_{i}\right) u(t) .
$$

It is reasonable to assume that the linearized model is accurate as long as the current state $T$ is not far away from the linearization point $T_{i}$ (i.e., $\left\|T-T_{i}\right\|<\delta$, where $\delta$ is a small number). Therefore, it is possible to approximate the nonlinear full-meshed system by combining a collection of the linear full-meshed models at different linearization points. Given $s$ linearized full-meshed models of the nonlinear system (13) which are expanded about states $T_{0}, T_{1}, \ldots, T_{s-1}$

$$
\begin{gathered}
\frac{\mathrm{d} T(t)}{\mathrm{d} t}=f\left(T_{i}\right)+A_{i}\left(T-T_{i}\right)+B\left(T_{i}\right) u(t), \\
\quad i=0,1, \ldots, s-1
\end{gathered}
$$

the nonlinear full-meshed system (10) can be approximated by a weighted combination of linear full-meshed models.

$$
\begin{aligned}
\frac{\mathrm{d} T}{\mathrm{~d} t}= & \sum_{i=0}^{i=s-1} \hat{w}_{i}(T)\left(f\left(T_{i}\right)+A_{i}\left(T-T_{i}\right)+B\left(T_{i}\right) u(t)\right) \\
& \times \sum_{i=0}^{i=s-1} \hat{w}_{i}(T)=1
\end{aligned}
$$

where $\hat{w}_{i}(T)$ is a normalized vector of state-dependent weights, which will be described in detail later.

Equation (15) is also called the trajectory piecewiselinear model (TPWL). Although equation (15) can represent the original nonlinear system for a certain trajectory of states, the system may be too large to be simulated simply because it requires many linear full-meshed models with different weightings at different states. In addition, generating the linearization points $T_{0}, T_{1}, \ldots, T_{s-1}$ usually requires a complete run of the nonlinear full-meshed model, which is also computationally expensive. In the following two subsections, we will introduce a model-order-reduction (MOR) procedure that will be used to create linearization points as well as low-order models.

\subsection{Generation of linearization points and order-reduction bases}

In this sub-section, we will describe the procedure of efficiently obtaining linearization points, and the procedure of constructing TPWL bases that will be used for creating reduced-order models. Note that the input current of the heat-transfer system is a function of $t$ (i.e., $J=J(t)$ and $\left.u(t) \propto J^{2}(t)\right)$, and that the temperature-dependent behavior of the electric resistivity (see equation (2)) is modeled in the $B$ matrix of equation (10). The procedure is as follows:

1. Set $i=0$.

2. Find the bases $V_{1}$ and $V_{2}$ :

the linearized model of the nonlinear system (equation (10)) about state $T_{i}$ can be obtained by rearranging equation (14):

$$
\frac{\mathrm{d} T}{\mathrm{~d} t}=A_{i} T+\left(f\left(T_{i}\right)-A_{i} T_{i}\right)+B\left(T_{i}\right) u(t) .
$$

Equation (16) can be considered as a linear system with two inputs (i.e., $u(t)$ and $\left.\left(f\left(T_{i}\right)-A_{i} T_{i}\right)\right)$, and therefore can be reduced by the block Arnoldi algorithm [10]. The corresponding bases $V_{1}$ and $V_{2}$ in the qth order Krylov subspaces can be obtained by using

$$
\begin{aligned}
\operatorname{span}\left\{V_{1}\right\} & =K_{q}\left(A_{i}^{-1}, b_{1}\right) \\
& =\operatorname{span}\left\{b_{1}, A_{i}^{-1} b_{1}, \ldots, A_{i}^{-(q-1)} b_{1}\right\}
\end{aligned}
$$

where $b_{1}=-A_{i}^{-1} B\left(T_{i}\right)$

$$
\begin{aligned}
\operatorname{span}\left\{V_{2}\right\} & =K_{q}\left(A_{i}^{-1}, b_{2}\right) \\
& =\operatorname{span}\left\{b_{2}, A_{i}^{-1} b_{2}, \ldots, A_{i}^{-(q-1)} b_{2}\right\},
\end{aligned}
$$

where $b_{2}=-A_{i}^{-1}\left(f\left(T_{i}\right)-A_{i} T_{i}\right)$.

3. Find the orthonormal basis $\hat{V}_{i}$ : the orthonormal basis $\hat{V}_{i}$ is obtained by taking the singular value decomposition (SVD) of the union of $V_{1}, V_{2}$ and $T_{i}$. It ensures that the reduced basis $\hat{V}_{i}$ will be able to 
represent the characteristics of the state $T_{i}$. With $\hat{V}_{i}$, the reduced-order system can be represented as

$$
\frac{\mathrm{d} T_{q i}}{\mathrm{~d} t}=A_{q i} T_{q i}+D_{q i}+B_{q i} u(t)
$$

where $A_{q i}=\hat{V}_{i}^{T} A_{i} \hat{V}_{i}, D_{q i}=\hat{V}_{i}^{T}\left(f\left(T_{i}\right)-A_{i} T_{i}\right), B_{q i}=$ $\hat{V}_{i}^{T} B\left(T_{i}\right)$ and $T_{q i}=\hat{V}_{i}^{T} T$.

4. If $i=0$, find parameters for estimating linearization points:

the linearized reduced-order dynamical system is performed to find the steady-state states $T_{f}$ and the steadystate time $t_{s}$. Although $t_{s}$ and $T_{f}$ is not accurate when compared with the results calculated by the original nonlinear full-meshed model, they provide the 'approximate' information about the steady-state time $t_{s}$, and the scale of changes between the initial and the final states, as shown below:

$$
d=\left\|T_{f}-T_{0}\right\| /\left\|T_{0}\right\| .
$$

The time incremental value for each time step can be set as $\Delta t=t_{s} / s$, where $s$ is the number of linearization points.

5. Set $t_{\mathrm{start}, i+1}=t_{\mathrm{start}, i}+\Delta t$ where $t_{\mathrm{star}, 0}=0$.

6. Simulate the reduced-order system until the next linearization point is found:

Simulate the linear reduced-order dynamical system (19) for subsequent $t_{\mathrm{start}, i}$ to $t_{\mathrm{start}, i+1}$.

i. If $\left\|\hat{V}_{i} T_{q i}\left(t_{\mathrm{start}, i+1}\right)-T_{i}\right\| /\left\|T_{i}\right\|<\alpha$, then $T_{i+1}=$ $\hat{V}_{i} T_{q i}\left(t_{\mathrm{start}, i+1}\right)$. Note that $\alpha$ is an appropriately selected constant. For most cases, $\alpha=d / s$ is a good choice.

ii. If $\left\|\hat{V}_{i} T_{q i}\left(t_{\mathrm{start}, i+1}\right)-T_{i}\right\| /\left\|T_{i}\right\|>\alpha$, then $\left\{t_{\mathrm{start}, i+1}=\right.$ $\left(t_{\mathrm{start}, i+1}+t_{\mathrm{star}, i}\right) / 2$; return to step 6$\}$.

7. Set $i:=i+1$; if $(i<s-1)$ return to step 2 .

8. Set $V=\operatorname{SVD}\left(\hat{V}_{0}, \hat{V}_{2}, \ldots, \hat{V}_{s-1}\right)$. Orthogonalize the union of $\hat{V}_{0}, \hat{V}_{2}, \ldots, \hat{V}_{s-1}$ using SVD and construct a new basis $V$.

Note that since the typical ranks of $A_{q i}, D_{q i}$ and $B_{q i}$ in equation (19) are very small, the computational cost for simulating the transient responses of equation (19) is usually trivial, so that the total cost of generating the basis $V$ is also trivial. Also note that the first $q$ moments of the transfer function of the reduced-order system in equation (19) are the same as the first $q$ moments of the transfer function of the system in equation (14) [18]. Therefore, equation (19) is potentially a good reduced model to approximate the dynamic behavior of the system described by equation (14) for each linearization point.

\subsection{Generating the TPWLMOR model}

In this sub-section, we will present the procedure of constructing TPWLMOR models using the collection of linearized full-meshed models (equation (15)) and the basis $V$ described in the previous sub-section. The procedure includes two steps, and is described as follows.
1. Construct the TPWLMOR model:

the process of constructing the TPWLMOR model is equivalent to replacing $T$ with $V T_{r}$ in equation (15).

$$
\begin{aligned}
\frac{\mathrm{d} T_{r}}{\mathrm{~d} t} & =\left(\sum_{i=0}^{s-1} w_{i}\left(T_{r}\right) A_{i r}\right) T_{r}+\gamma \cdot w\left(T_{r}\right) \\
& +\left(\sum_{i=0}^{s-1} w_{i}\left(T_{r}\right) B_{i r}\right) u(t)
\end{aligned}
$$

where $A_{i r}=V^{T} A_{i} V, \quad B_{i r}=V^{T} B_{i}$ and $\gamma=$ $\left[V^{T}\left(f\left(T_{0}\right)-A_{0} T_{0}\right), \ldots, V^{T}\left(f\left(T_{s-1}\right)-A_{s-1} T_{s-1}\right)\right]$.

2. Compute the weighting at each time step during a transient simulation.

i. For $i=0, \ldots,(s-1)$ compute $d_{i}=\left\|T-T_{i}\right\|_{2}$.

Note that the smaller the $d_{i}$, the smaller the distance between $T$ and $T_{i}$. Also, the distance between $T$ and $T_{i}$ is equal to the distance between the orthonormal projections of $T$ and $T_{i}$, which is shown below:

$$
\begin{aligned}
\| T_{r} & -V^{T} T_{i}\left\|_{2}=\right\| V^{T} T-V^{T} T_{i} \|_{2} \\
& =\left(\left(T-T_{i}\right) V^{T} V\left(T-T_{i}\right)\right)=\left\|T-T_{i}\right\|_{2} .
\end{aligned}
$$

ii. Take $m=\min _{i=0}\left(d_{i}\right)$.

iii. For $i=0, \ldots,(s-1)$ compute $\tilde{w}_{i}=\mathrm{e}^{-\lambda d_{i} / m}$.

iv. Normalize $\tilde{w}_{i}$

$$
\text { For } i=0, \ldots,(s-1) \text { set } w_{i}\left(T_{r}\right)=\frac{\tilde{w}_{i}\left(T_{r}\right)}{\sum_{j=0}^{s-1} \tilde{w}_{j}\left(T_{r}\right)} \text {. }
$$

These two steps are computed for each time step during the time integration of a transient simulation. We use an implicit scheme for the time integration. With the weighting $w_{i}\left(T_{r}\right)$ calculated in step 2, equation (21) ensures that the TPWLMOR model rapidly reduces to a certain linearized model for most of the time. From our preliminary study, the suggested values for $\lambda$ (in equation 23) are about 20-25. Figure 1 summarizes the macromodeling procedures described in this section.

\section{TPWLMOR case studies}

In this section, two examples of nonlinear MEMS heattransfer macromodeling using the TPWLMOR technique are presented. The first example explores the characteristics of a thermal flexure actuator [1]. The second example demonstrates the temperature calculation for a thermal in-plane microactuator [16]. The study of the accuracy versus the orders of TPWLMOR models is also discussed.

\subsection{Case study I: thermal flexure actuator}

Figure 2 shows a CCD picture of a thermal actuator which is fabricated by the multi-user MEMS process (MUMPS) [19]. The detailed operational principle can be found in [8]. Natural convection and radiation boundary conditions are applied to the surfaces adjacent to the air. The temperature of the interfaces between the anchors and the substrate is fixed at $300 \mathrm{~K}$. The dimensions of the thermal actuator are tabulated in table 2. The thermal actuator is meshed into 2835 nodes.

The simulated results in this case study are calculated by the following three different types of models: 


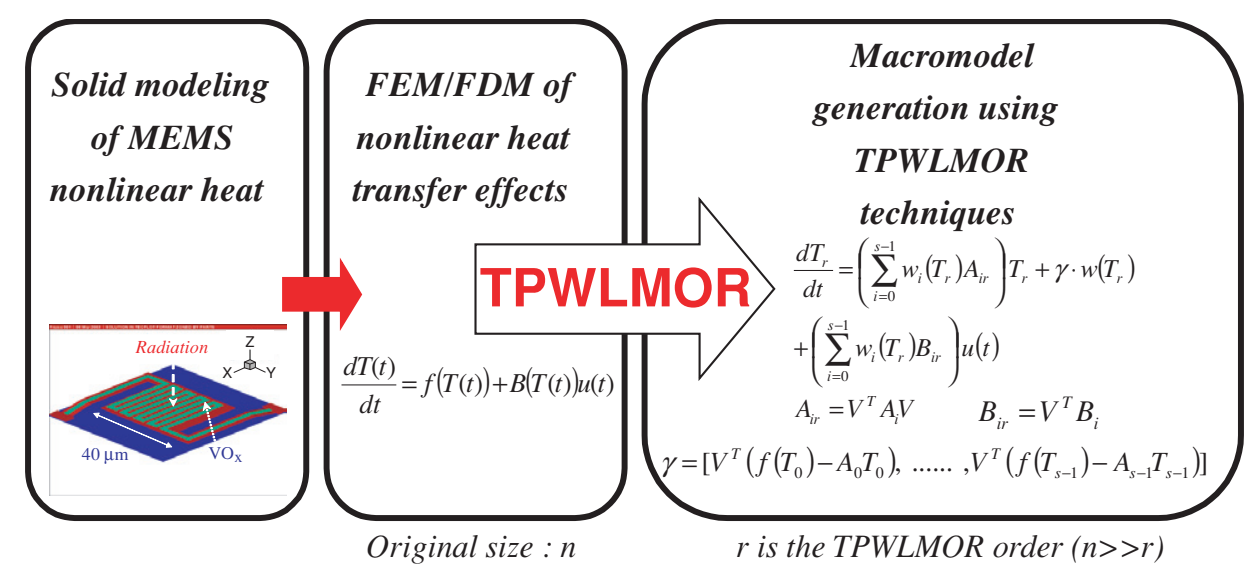

(a)

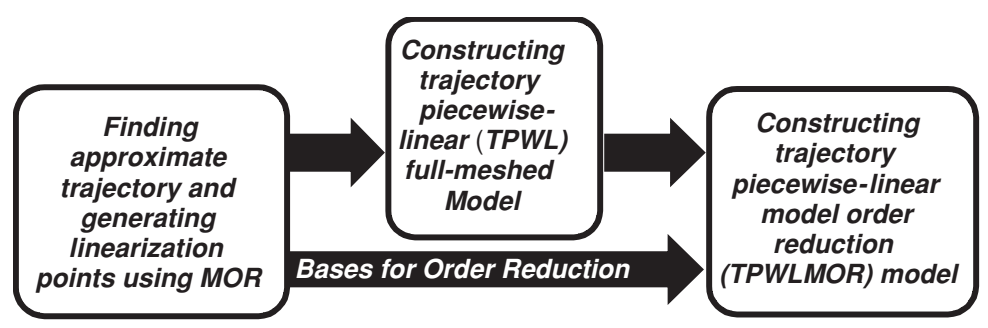

(b)

Figure 1. (a) The procedure of macromodeling the nonlinear heat-transfer problem using the TPWLMOR algorithm. $(b)$ The procedure of the TPWLMOR algorithm.

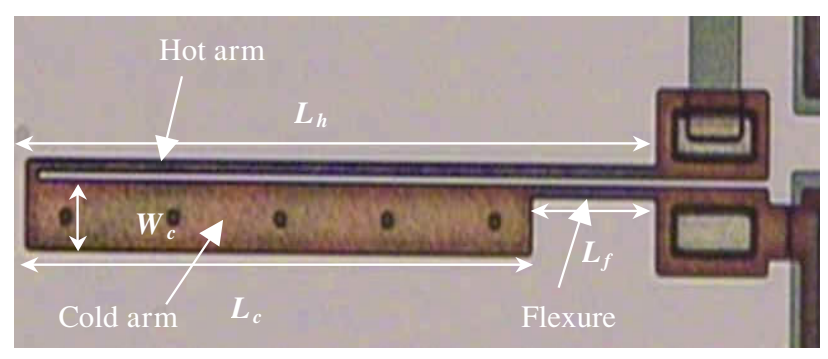

Figure 2. A CCD picture of the thermal device analyzed and measured in this work. The contact pads are on the right side of the picture and are not completely shown.

Table 2. The dimensions of the thermal actuator.

\begin{tabular}{ll}
\hline Geometrical dimensions & Length $(\mu \mathrm{m})$ \\
\hline Length of hot arm $\left(L_{h}\right)$ & 200 \\
Width of hot arm $\left(W_{h}\right)$ & 3 \\
Length of cold arm $\left(L_{c}\right)$ & 160 \\
Width of cold arm $\left(W_{c}\right)$ & 16 \\
Gap $(g)$ & 3 \\
Length of flexure $\left(L_{f}\right)$ & 40 \\
Width of flexure $\left(W_{f}\right)$ & 3 \\
Thickness $(t)$ & 2 \\
\hline
\end{tabular}

(1) Linear full-meshed models. In this type of model, all the material properties are assumed to be constants (the values at $300 \mathrm{~K}$ ). The radiation effect is also neglected. Note that this type of model is similar to the full-meshed models used in $[7,8]$.

(2) Nonlinear full-meshed model. In this type of model, the temperature-dependent material properties as well as the radiation effects are considered.
Table 3. The material properties of the thermal actuator in the linear full-meshed case.

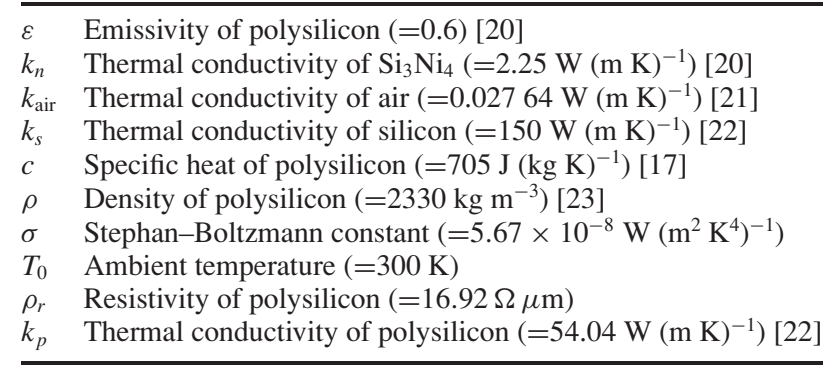

(3) TPWLMOR models. The TPWLMOR models are generated by reducing the nonlinear full-meshed models using the TPWLMOR methodology presented in this work.

The material properties for this case study are listed in tables 3 and 4 . Figure 3 shows the average temperatures versus time for a $4.5 \mathrm{~mA}$ input calculated by different types of models. The results calculated by the TPWLMOR model follow the results of the nonlinear full-meshed model very well. Figure 4 shows the mean square relative difference (MSRD) for all nodes between the nonlinear full-meshed model and the TPWLMOR models with various orders at $4.5 \mathrm{~mA}$ and $s=10$. The MSRD is defined as

$$
\operatorname{MSRD}(t)=\sqrt{\frac{\sum_{j=1}^{n}\left(\frac{T_{j}(t)-\hat{T}_{j}(t)}{T_{j}(t)}\right)^{2}}{n}}
$$

where $T_{j}(t)$ is the $j$ th node temperature calculated by the nonlinear full-meshed model over time, $\hat{T}_{j}(t)=\left(V T_{r}(t)\right)_{j}$ is 
Nonlinear heat-transfer macromodeling for MEMS thermal devices

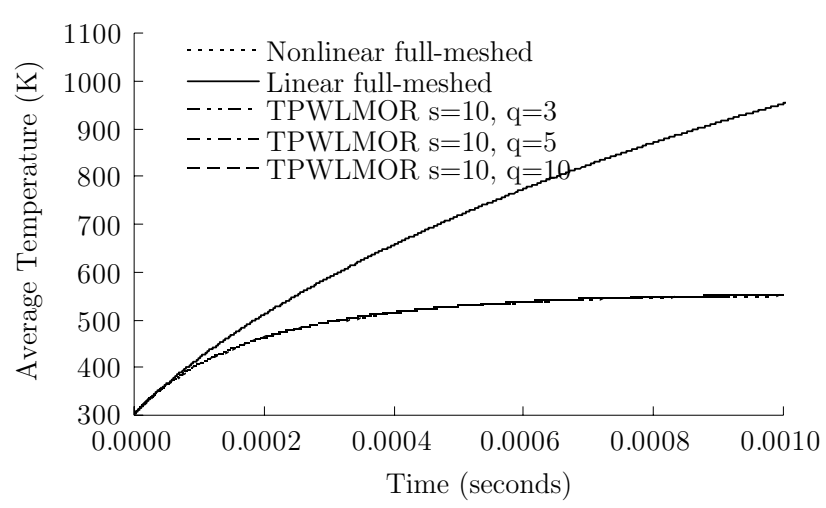

Figure 3. Comparison of the results by the nonlinear full-meshed model, the linear full-meshed model and the TPWLMOR models for the average temperature of the thermal actuator with an input current of $4.5 \mathrm{~mA}$.

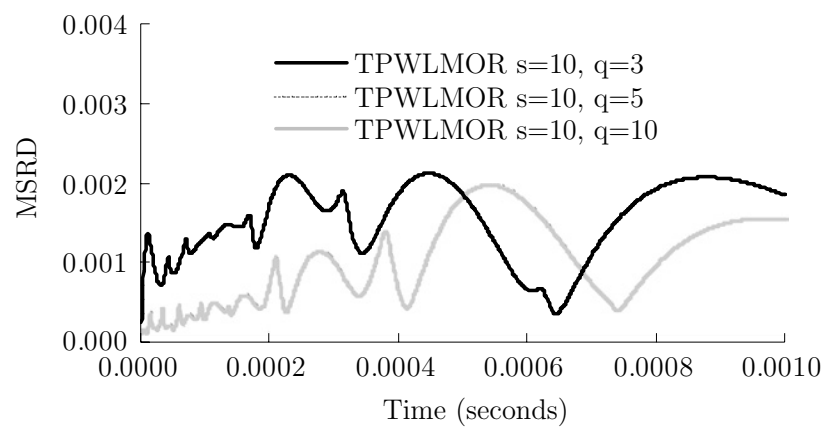

Figure 4. The MSRD between the full-meshed nonlinear model and the TPWLMOR models with various orders at $4.5 \mathrm{~mA}$ and $s=10$.

Table 4. The material properties of the thermal actuator in the nonlinear full-meshed case and the TPWLMOR case.

\begin{tabular}{ll}
\hline$k_{\text {air }}$ & Temperature-dependent conductivity of air $(=9.97 \times$ \\
& $\left.10^{-3}+5.89 \times 10^{-5} T \mathrm{~W}(\mathrm{~m} \mathrm{~K})^{-1}\right)[21]$ \\
$\rho_{r}(T) \quad \begin{array}{l}\text { Temperature-dependent resistivity of polysilicon }\left(=\rho_{0}[1+\right. \\
\end{array}$ & $\left.\beta\left(T-T_{0}\right)\right]$, where $\rho_{0}=16.92 \Omega \mu \mathrm{m}$ and $\beta=1.25 \times$ \\
& $\left.10^{-3} \mathrm{~K}^{-1}\right)[24]$ \\
$k_{p}(T) \quad \begin{array}{l}\text { Temperature-dependent thermal conductivity of polysilicon } \\
\left(=\left[\left(-2.2 \times 10^{-11}\right) T^{3}+\left(9.0 \times 10^{-8}\right) T^{2}+(-1.0 \times\right.\right. \\
\\
\left.\left.\left.10^{-5}\right) T+0.014\right]^{-1} \mathrm{~W}(\mathrm{~m} \mathrm{~K})^{-1}\right)[22]\end{array}$ \\
\hline
\end{tabular}

Table 5. The performance comparison of the nonlinear full-meshed model and the TPWLMOR models in the thermal actuator case study.

\begin{tabular}{lcc}
\hline & Simulation time (s) & Speed-up factor \\
\hline Nonlinear full-meshed & 45603.79 & 1 \\
TPWLMOR, $s=10, q=3$ & 155.56 & 293.16 \\
TPWLMOR, $s=10, q=5$ & 182.32 & 250.13 \\
TPWLMOR, $s=10, q=10$ & 367.00 & 124.26 \\
\hline
\end{tabular}

the temperature of the $j$ th node calculated by the TPWLMOR model and $n$ is the total number of nodes. For the TPWLMOR model with $s=10$ and $q=3$, the MSRD is less than $0.3 \%$.

Table 5 presents the comparison of the performance between the nonlinear full-meshed model and the TPWLMOR models with various orders. The results demonstrate that the TPWLMOR models with an order equal to 3 (or greater than $3, q \geqslant 3$ ) give a 2 order-of-magnitude reduction in
Table 6. The generation times of the TPWLMOR models at $4.5 \mathrm{~mA}$ for the thermal flexure actuator.

\begin{tabular}{ll}
\hline TPWLMOR parameters & Model generation time (s) \\
\hline$s=5, q=3$ & 8.22 \\
$s=5, q=5$ & 13.25 \\
$s=5, q=10$ & 27.58 \\
$s=8, q=3$ & 12.36 \\
$s=8, q=5$ & 20.5 \\
$s=8, q=10$ & 42.21 \\
$s=10, q=3$ & 15.36 \\
$s=10, q=5$ & 25.63 \\
$s=10, q=10$ & 52.84 \\
$s=15, q=3$ & 24.51 \\
$s=15, q=5$ & 39.42 \\
$s=15, q=10$ & 82.48 \\
\hline
\end{tabular}

computational times. All the simulations are performed using a PC with a $2.8 \mathrm{GHz}$ xeon CPU and $1024 \mathrm{MB}$ RAM. The TPWLMOR model-generation times (i.e., the computational times required for the first two steps in figure $1(b)$ ) for different $s$ and $q$ are listed in table 6 . These model-generation times are insignificant compared with the computational times required by the nonlinear full-meshed models and the TPWLMOR models.

Figure 5 shows the simulated transient average temperatures of the hot arm, the cold arm and the flexure when an input current of $4.5 \mathrm{~mA}$ is applied. Compared with the nonlinear models, the linear full-meshed model overpredicts the temperatures. The deflection of the thermal actuator can be evaluated by a thermomechanical closed-form model proposed by $[8,20]$. This approach of evaluating deflection works rather well because the deformations of structure calculated by the thermomechanical closed-form model rarely affect the temperature solution originally calculated by electrothermal solvers (i.e., weakly coupled problems). Since the closed-form model for evaluating the deflection is lengthy and its derivation is quite tedious, it is not described in this paper. Note that the nonlinear thermomechanical effect (temperature dependence of the thermal expansion coefficient) is also considered in [8].

The simulated transient responses of the tip deflection at $4.5 \mathrm{~mA}$ are shown in figure 6 . The deflection reaches a maximum value at about $0.2 \mathrm{~ms}$, and then slowly decreases to a steady value. This interesting behavior arises from the fact that the thermal time constant of the hot arm is shorter than that of the cold arm, as shown in figure 5. The maximum deflection occurs when the average temperature of the hot arm reaches steady state (maximum). Then the deflection decreases slowly since the average temperature of the cold arm is still increasing. The deflection reaches a steady state as the average temperature of the device becomes steady. The measured tip deflections of the thermal actuator are also presented. Figure 7 shows the tip displacements versus input currents from $1 \mathrm{~mA}$ to $4.5 \mathrm{~mA}$. The error bars represent 1 standard deviation. The measured and the calculated results are in good agreement. Table 7 lists the simulated deflections by various models at different input currents.

In order to study the transient characteristics of the device, we measure the transient voltages of the thermal actuator under constant current inputs. A step current is applied to the actuator by using a source meter (Keithley 2400), and the corresponding 


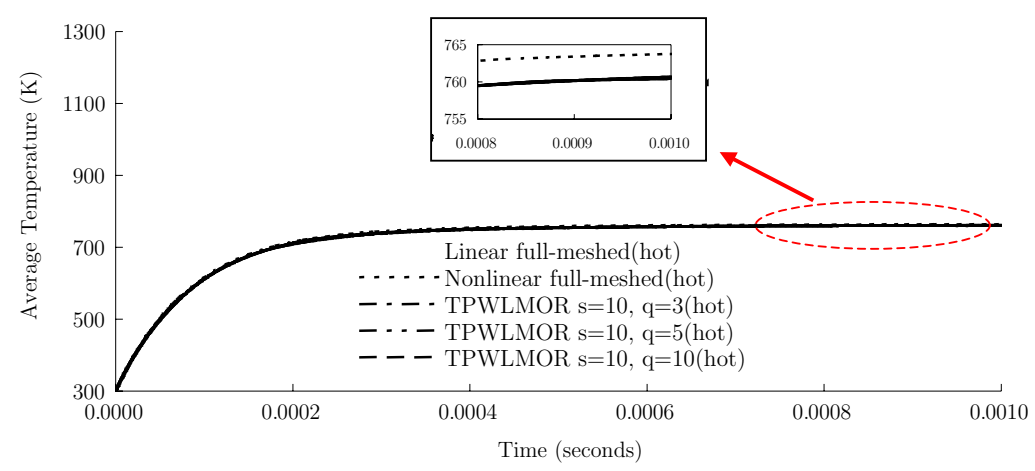

(a)

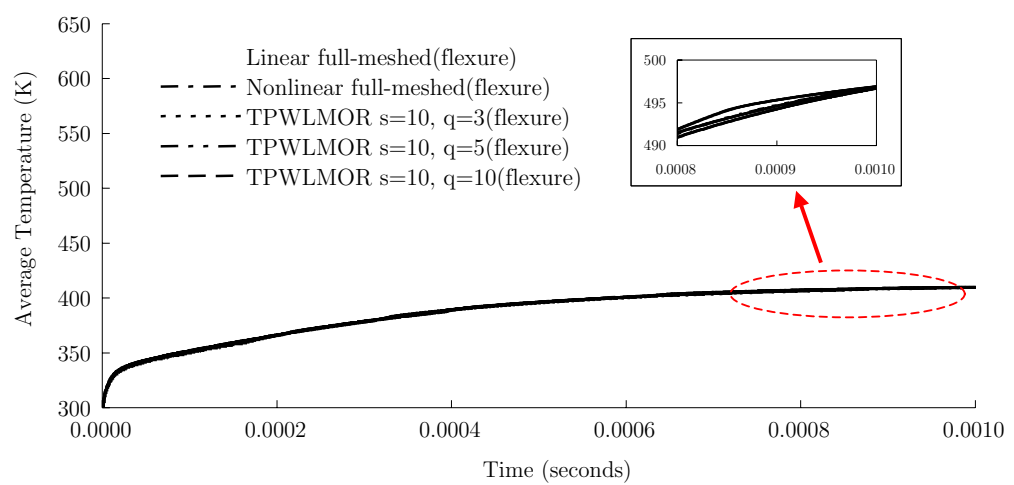

(b)

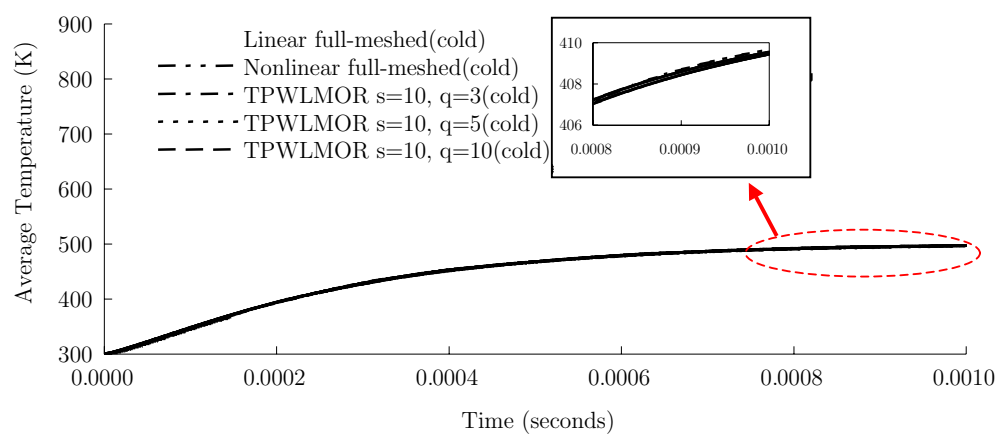

(c)

Figure 5. The average temperature of $(a)$ the hot arm, $(b)$ the cold arm and $(c)$ the flexure calculated by the TPWLMOR models and the linear/nonlinear full-meshed models. These calculated temperatures are used for estimating the tip deflections of the thermal actuator.

Table 7. A comparison of the simulated voltages and deflections by various models at different input currents for the thermal flexure actuator.

\begin{tabular}{llllllll}
\hline & \multicolumn{3}{l}{$\begin{array}{l}\text { Voltages at different } \\
\text { input currents }(V)\end{array}$} & & \multicolumn{3}{l}{$\begin{array}{l}\text { Deformations at different } \\
\text { input currents }(\mu \mathrm{m})\end{array}$} \\
\cline { 2 - 3 } \cline { 7 - 9 } & $2 \mathrm{~mA}$ & $3 \mathrm{~mA}$ & $4 \mathrm{~mA}$ & & $2 \mathrm{~mA}$ & $3 \mathrm{~mA}$ & $4 \mathrm{~mA}$ \\
\hline Linear full-meshed & 1.5400 & 2.3100 & 3.0800 & & 1.8701 & 4.2077 & 7.4803 \\
Nonlinear full-meshed & 1.6524 & 2.7264 & 4.1852 & & 1.1378 & 2.9957 & 6.4127 \\
TPWLMOR, $s=10, q=3$ & 1.6512 & 2.7240 & 4.1748 & & 1.1423 & 2.9791 & 6.4186 \\
TPWLMOR, $s=10, q=5$ & 1.6513 & 2.7241 & 4.1743 & & 1.1411 & 2.9792 & 6.4186 \\
TPWLMOR, $s=10, q=10$ & 1.6513 & 2.7242 & 4.1743 & & 1.1411 & 2.9811 & 6.4188 \\
\hline
\end{tabular}

transient voltage drop across the actuator is measured by an oscilloscope. The resistance variation of the device can be estimated by the calculated temperature distribution in the device, and thus the voltage across the device can be evaluated accordingly.
Figure 8 shows the simulated and the measured transient voltages. The measured curve is the average of 12 measurement results. The error bars, which are indicated on the curve every $0.05 \mathrm{~ms}$, represent 1 standard deviation. Since the resistance in the hot arm is much larger than those 


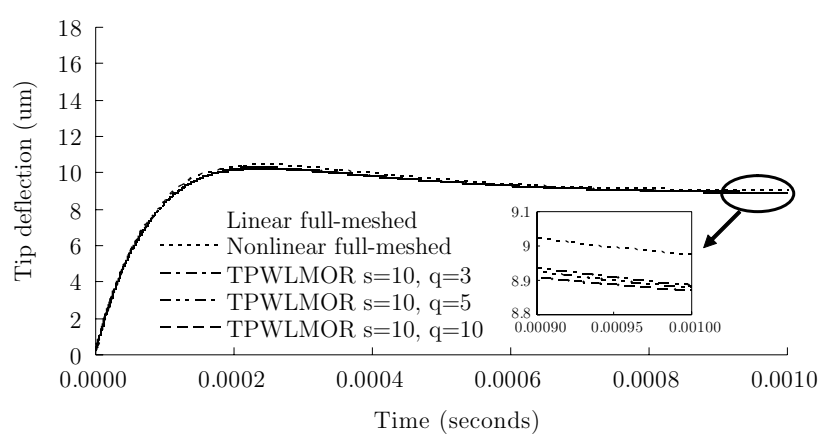

Figure 6. Comparison of the transient tip deflection calculated by the TPWLMOR models and the linear/nonlinear full-meshed models when the input current is $4.5 \mathrm{~mA}$.

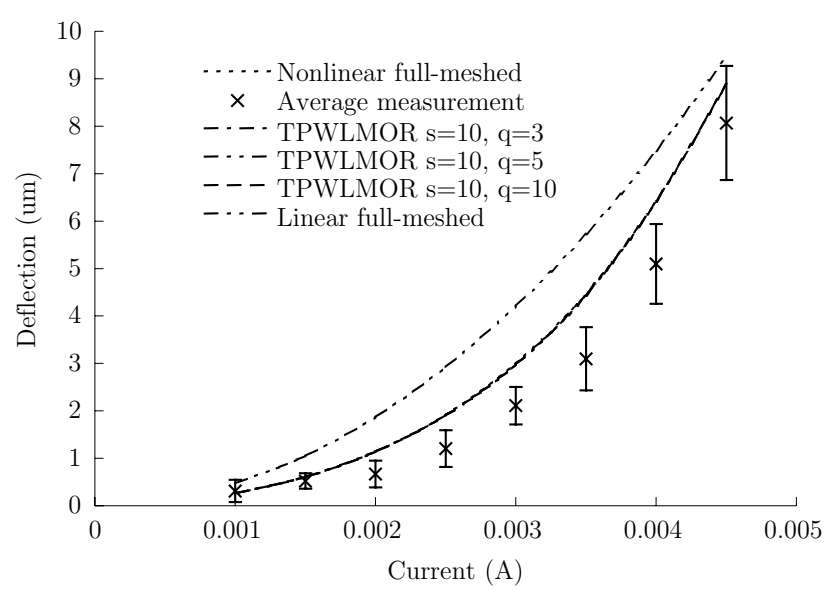

Figure 7. Comparison of the measured and calculated tip displacements with input currents from $1 \mathrm{~mA}$ to $4.5 \mathrm{~mA}$.

in the cold arm and the flexure, most of the voltage drop across the device exists in the hot arm. Therefore, as the temperature increases, the hot arm contributes the largest increase of resistance of the device. As a result, the transient behavior of voltage should be closely correlated with the transient behavior of temperature in the hot arm. This can be observed by the fact that the time constant shown in figure 8 is very close to the time constant in figure $5(a)$. Also, in figure 6 , the time to reach the maximum tip deflection

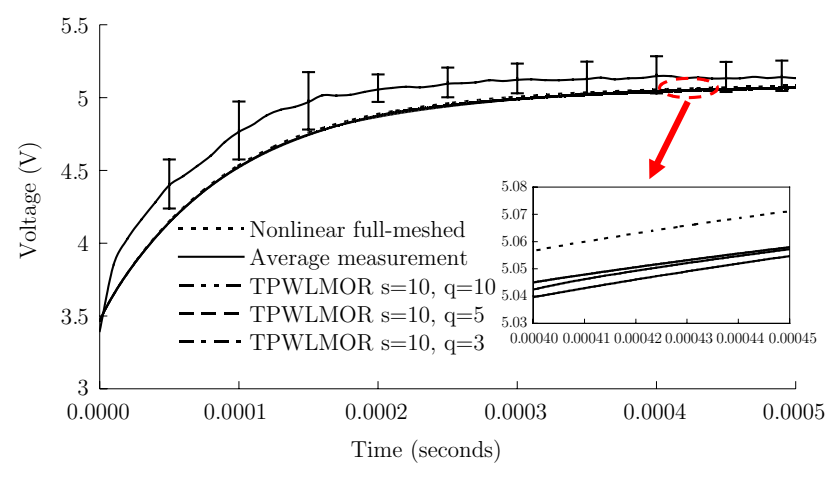

Figure 8. A comparison of transient voltages between measured and simulated results with an input current of $4.5 \mathrm{~mA}$.

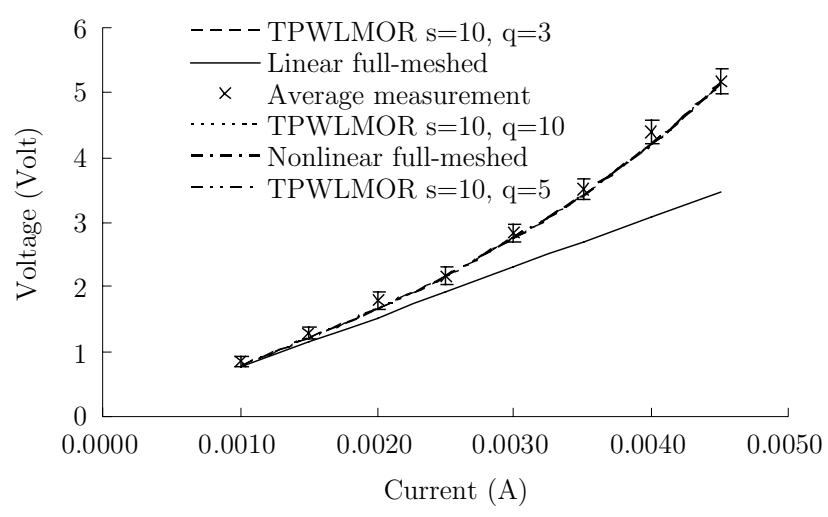

Figure 9. A comparison of steady-state voltages between measured and simulated results from $1 \mathrm{~mA}$ to $4.5 \mathrm{~mA}$.

(at about $0.2 \mathrm{~ms}$ ) is also consistent with the time when the voltage approaches the steady state, as shown in figure 8 . These results indicate that the TPWLMOR model is not only capable of accurately predicting steady behavior, but also capable of capturing transient thermal effects very well.

Figure 9 shows the comparison between the simulated and measured steady-state voltages under different input currents. The discrepancies are negligible (less than $0.1 \%$ ) between the simulated results by the TPWLMOR models and by the nonlinear full-meshed model. The measured results are also in very good agreement with the simulated results. Table 7 lists the simulated voltages by various models at different

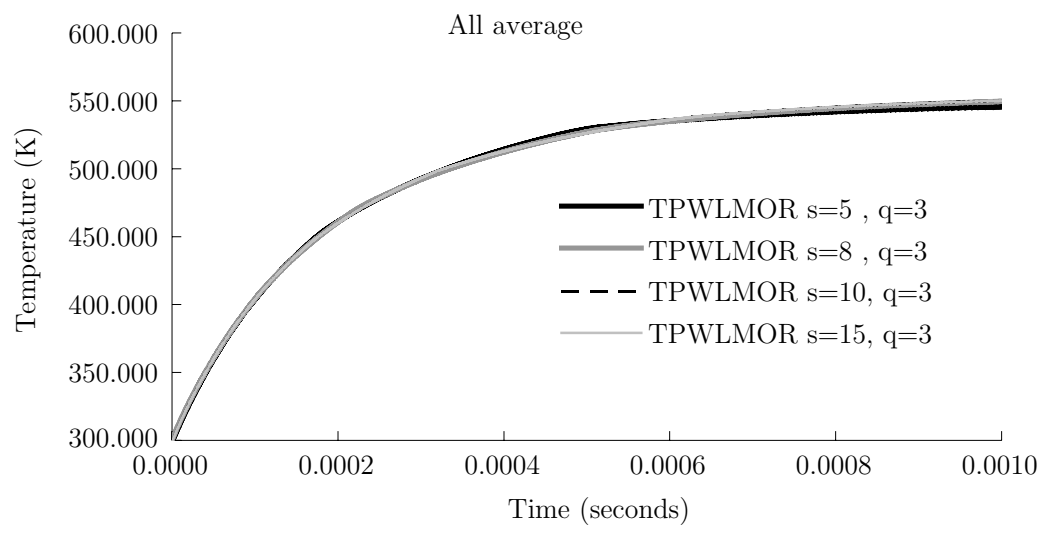

Figure 10. A comparison of average temperature of different numbers of linearization points $(s)$ at $4.5 \mathrm{~mA}$ input and $q=3$. 


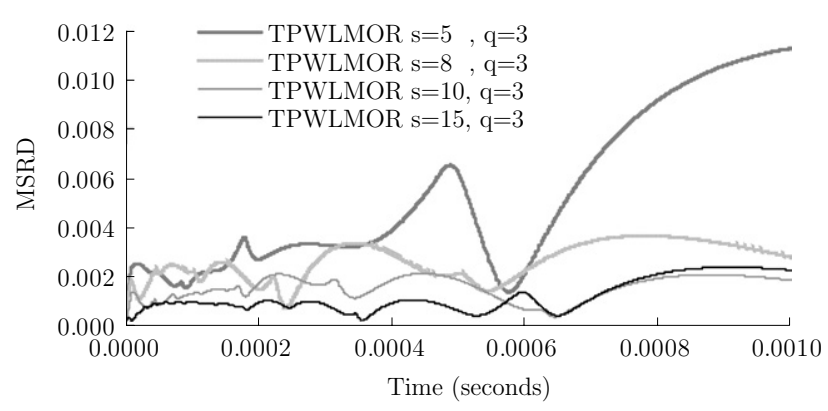

Figure 11. The MSRD of different numbers of linearization points $(s)$ at $4.5 \mathrm{~mA}$ input and $q=3$.

input currents. The voltages simulated by the linear fullmeshed model are lower than the measured results because the linear full-meshed model cannot account for the increase of resistivity due to a temperature increase.

Figure 10 shows the transient average temperatures at $4.5 \mathrm{~mA}$ input current with different numbers of linearization points $(s)$. Note that the order $(q)$ is 3 for these simulated cases. Figure 11 shows the MSRD versus time for different $s$ at $4.5 \mathrm{~mA}$ input. From the figures, it is clear that the error is negligible (less than $0.3 \%$ ) if $s$ is greater than 8 .

We also demonstrate the simulated results of the actuator with a sinusoidal current input. The applied time-dependent current input is

$$
i=2(1-\cos (2 \pi f t)) \mathrm{mA}
$$

where the frequency $f$ is $10000 \mathrm{~Hz}$. Figure 12 shows the calculated average temperatures of the actuator. The results by the TPWLMOR models and by the nonlinear full-meshed model are in very good agreement. The MSRDs with various orders are shown in figure 13. It is observed that for the TPWLMOR model with order $3(q=3)$, the maximum MSRD is less than $0.5 \%$.

\subsection{Case study II: TIM microactuator}

In this sub-section, we will demonstrate the TPWLMOR modeling results for a thermal in-plane microactuator (TIM) [16] fabricated by the MUMPS process. Figure 14 shows the schematic view of the TIM. The TIM consists of a suspended shuttle which is connected to electrical contact pads by slightly inclined slender legs. The electric current flowing through the structure results in thermal deformation in the slender legs, which in turn causes the in-plane motion of the shuttle. Since the behavior of a single leg-pair is equivalent to the behavior of the full TIM device [16], in this work, we only focus on the modeling of a single leg-pair. The material properties used for the TIM simulation are the same as those used for the first case study, except that $T_{0}$ is $293 \mathrm{~K}$ and $\rho_{0}$ is $34 \Omega \mu \mathrm{m}$. Detailed descriptions of the boundary conditions, the (temperaturedependent) material properties and the dimensions of the TIM can be found in [16].

The 3D FDM model of the single leg-pair of the TIM has 2196 nodes. Figure 15 shows the temperature profiles at $5 \mathrm{~mA}$ input calculated by various models. Note that the thermal conductivity of the air surrounding the structure is also considered in this case. Figure 16 shows transient average temperatures of the TIM's legs calculated by the nonlinear fullmeshed model and the TPWLMOR models of different orders.

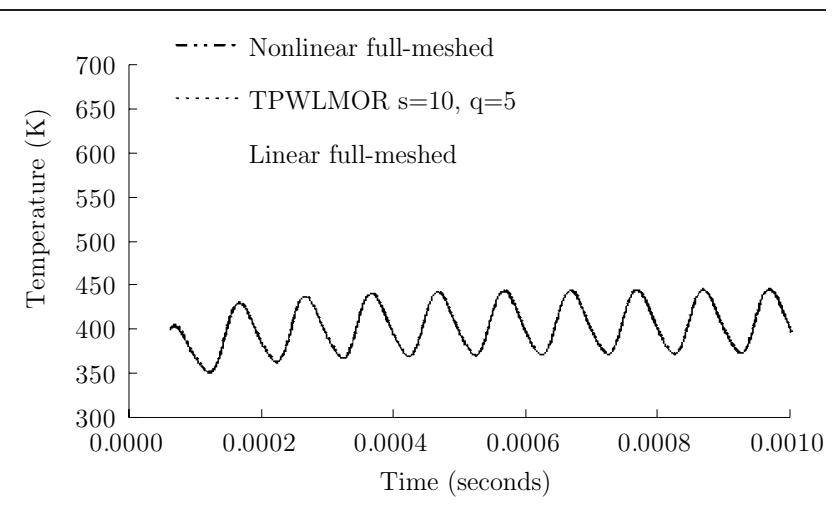

(a)

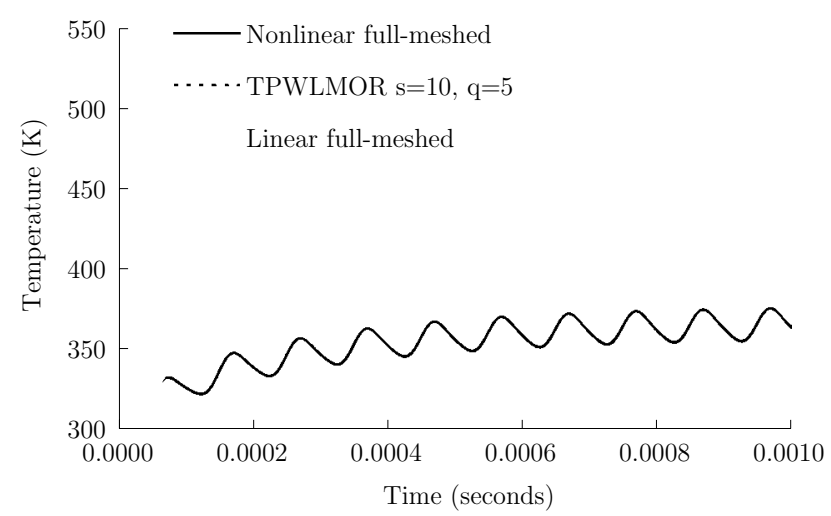

(b)

Figure 12. The average temperature of $(a)$ the hot arm, $(b)$ the cold arm, $(c)$ the flexure and $(d)$ the whole device calculated by the TPWLMOR models and nonlinear full-meshed model at input current $2(1-\cos (2 \pi f))$ and $s=10$.

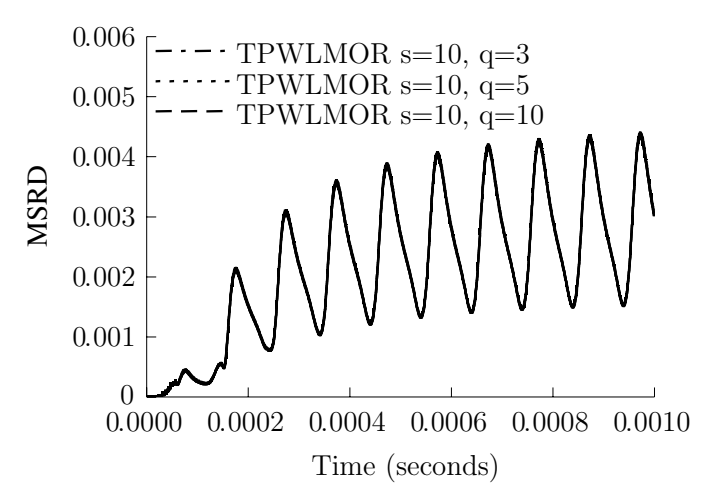

Figure 13. The MSRD between the nonlinear full-meshed model and TPWLMOR models with various orders at input current $2(1-\cos (2 \pi f))$ and $s=10$.

The MSRDs between the nonlinear full-meshed model and the TPWLMOR models are less than $0.3 \%$. Table 8 lists the computational costs of the nonlinear full-meshed model and the TPWLMOR models. Similar to the previous case study, the TPWLMOR models give 2 order-of-magnitude reductions in computational times. Note that the steady and transient results calculated by our models (figures 15 and 16) follow the simulated results of Lott et al [16] very well. It has to be emphasized that in [16] their simulated results were verified with experimentally measured results, which in turn justifies the results calculated by the models presented in this work. 


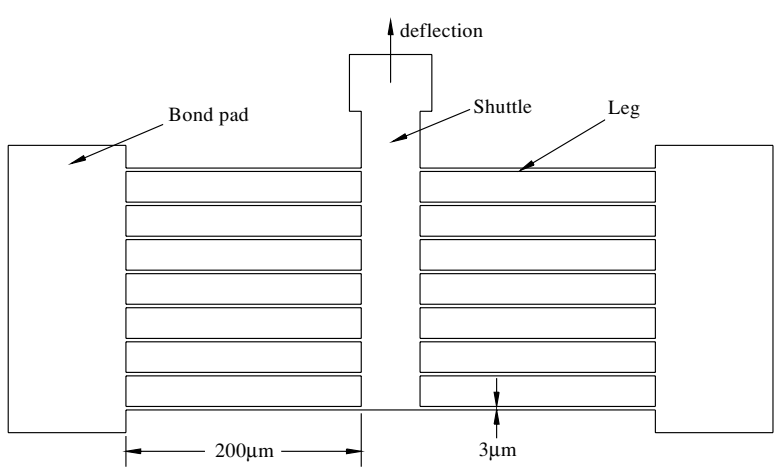

(a)

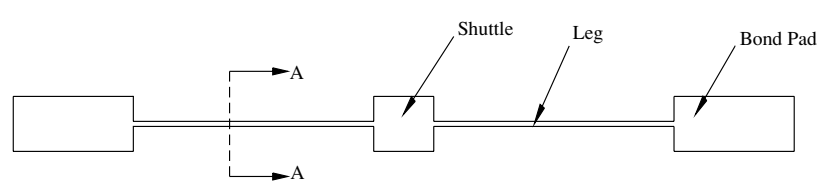

(b)

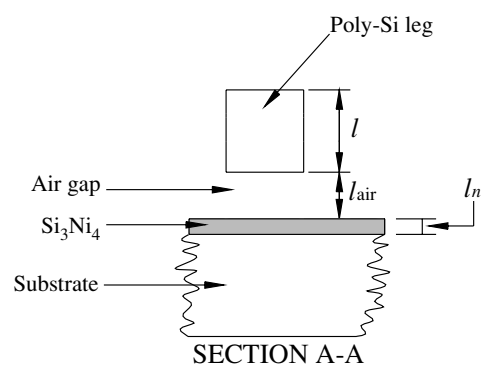

(c)

Figure 14. The schematic of the thermomechanical in-plane microactuator (TIM) [16]. (a) The top view of TIM, (b) the schematic of single legleg-pair and $(c)$ the A-A cross-section view of a leg.

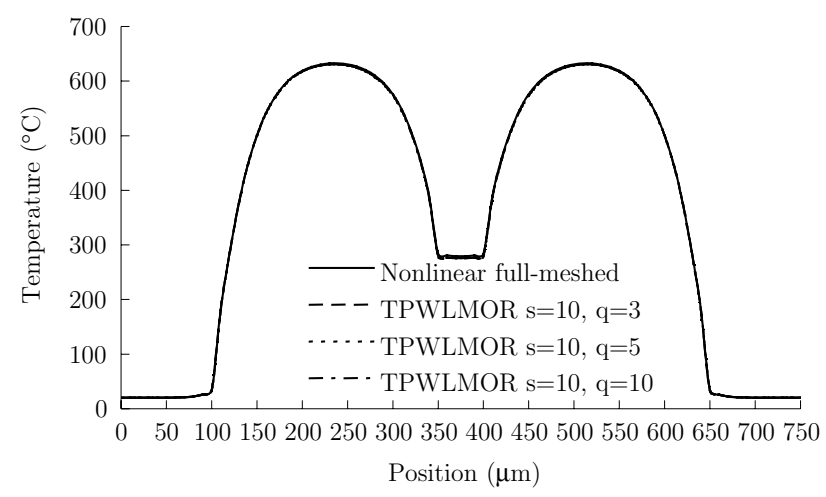

Figure 15. The calculated temperature profiles at $5 \mathrm{~mA}$ input with the nonlinear full-mesh model and TPWLMOR models with various orders with $s=10$.

Table 8. A performance comparison of the nonlinear full-meshed model and the TPWLMOR models in the TIM case study.

\begin{tabular}{|c|c|c|}
\hline & Simulation time (s) & Speed-up factor \\
\hline Nonlinear full-meshed & 33840 & 1 \\
\hline TPWLMOR, $s=10, q=3$ & 195.03 & 173.51 \\
\hline TPWLMOR, $s=10, q=5$ & 398.32 & 84.96 \\
\hline TPWLMOR, $s=10, q=10$ & 425.51 & 79.53 \\
\hline
\end{tabular}

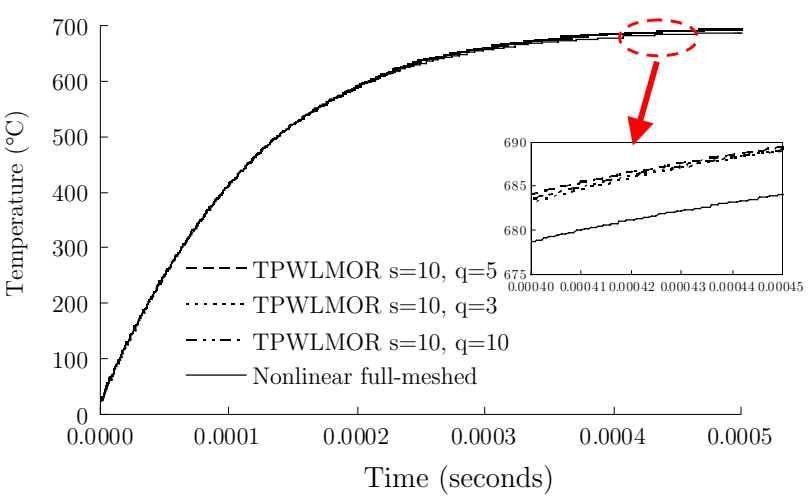

Figure 16. The calculated transient average temperature of TIM legs at $5.7 \mathrm{~mA}$ input with the nonlinear full-mesh model and TPWLMOR models with various orders with $s=10$.

\section{Conclusion}

A trajectory piecewise-linear model-order-reduction (TPWLMOR) technique for generating nonlinear MEMS heat-transfer compact models is presented. The TPWLMOR method uses a model-order-reduction algorithm as well as a piecewise-linear approximation to create nonlinear compact models. The procedure of the TPWLMOR method can be summarized in three steps: (1) searching for linearization points using the linear reduced-order model, (2) creating the piecewise-linear model which is a weighted combination of the linearized full-meshed models and (3) generating the compact model using the model-order-reduction algorithm. Two MEMS thermal devices with nonlinear effects are modeled using the TPWLMOR method. The nonlinearity effects include the temperature-dependent material properties and radiation effect. The heat conduction from the suspended structures to the substrate through the air film is also taken into consideration for more accurate modeling.

The simulated steady-state and transient results of the TPWLMOR models match the results of the nonlinear fullmeshed models very well (less than $0.5 \%$ discrepancy for each case). We also show that the measured steady-state results, which include the actuator tip deflection and the voltage drop through the structure, agree with the simulated results of the TPWLMOR models. The simulated transient behaviors also match the measured data. Therefore, the TPWLMOR models are not only capable of accurately predicting steadystate behavior, but also capable of capturing transient thermal effects. The computational costs of TPWLMOR models are about 2 orders of magnitude more efficient than those of the original full-meshed models. We also demonstrate that when the number of linearization points is equal to or larger than 8 $(s \geqslant 8)$, the errors between TPWLMOR models and nonlinear full-meshed models are negligible. Finally, the simulated and measured results also indicate that considerations of the temperature-dependent material properties are essential for accurate thermal modeling.

\section{Acknowledgments}

This work is supported by the National Science Council of Taiwan, Republic of China, under contract number NSC 922213-E-002-083. 


\section{References}

[1] Comtois J H and Bright V M 1996 Surface micromachined polysilicon thermal actuator arrays and applications Technical Digest of 1996 Solid-State Sensor and Actuator Workshop (Hilton Head Island, SC, June 1996) pp 174-7

[2] Cole B E, Higashi R E and Wood R A 1998 Monolithic two-dimensional arrays of micromachined microstructures for infrared applications Proc. IEEE 86 1679-86

[3] Amon C H, Murthy J Y, Yao S C, Narumanchi S, Wu C-F and Hsieh C-C 2001 MEMS-enabled thermal management of high-heat-flux devices, edifice: embedded droplet impingement for integrated cooling of electronics Exp. Therm. Fluid Sci. 25 231-42

[4] Daniel J H, Iqbal S, Millington R B, Moore D F, Lowe C R, Leslie D L, Lee M A and Pearce M J 1998 Silicon microchambers for DNA amplification Sensors Actuators A 71 81-8

[5] Senturia S D 2001 Microsystem Design (Boston: Kluwer)

[6] Yang Y-J, Gretillat M and Senturia S D 1997 Effect of air damping on the dynamics of nonuniform deformations of microstructures Proc. Transducers' 97 (Chicago, June 1997) pp 1093-6

[7] Bechtold T, Rudnyi E B and Korvink J G 2003 Automatic generation of compact electro-thermal models for semiconductor devices Proc. IEICE Trans. Electron. E86-C 459-65

[8] Yang Y J and Yu C C 2004 Extraction of heat-transfer macromodels for MEMS devices J. Micromech. Microeng. 14 587-96

[9] Yang Y-J, Kamon M, Rabinovich V L, Ghaddar C, Deshpande M, Greiner K and Gilbert J R 2001 Modeling gas damping and spring phenomena in MEMS with frequency dependent macro-models Proc. IEEE MEMS (Interlaken, Switzerland, January 2001) pp 365-8

[10] Odabasioglu A, Celik M and Pileggi L T 1998 PRIMA: passive reduced-order interconnect macromodeling algorithm IEEE Trans. Comput.-Aided Des. Integr. Circuits Syst. 17 645-54
[11] Hung E and Senturia S D 1999 Generating efficient dynamical models for microelectromechanical systems from a few finite-element simulation runs J. Microelectromech. Syst. 8 280-9

[12] Yang Y-J, Cheng S-Y and Shen K-Y 2004 Macromodeling of coupled-domain MEMS devices with electrostatic and electrothermal actuations J. Micromech. Microeng. 14 1190-6

[13] Rewieński M and White J 2003 A trajectory piecewise-linear approach to model order reduction and fast simulation of nonlinear circuits and micromachined devices IEEE Trans. Comput.-Aided Des. Integr. Circuits Syst. 22 155-70

[14] Rewieński M 2003 A trajectory piecewise-linear approach to model order reduction of nonlinear dynamical systems PhD Dissertation, Massachusetts Institute of Technology, Cambridge, MA

[15] Mills A F 1992 Heat Transfer (Boston: Richard D Irwin Inc.)

[16] Lott C D, McLain T W, Harb J N and Howell L L 2002 Modeling the thermal behavior of a surface-micromachined linear-displacement thermomechanical microactuator Sensors Actuators A $10181-8$

[17] Lin L-W and Chiao M 1996 Electrothermal responses of lineshape microstructures Sensors Actuators A 55 $35-41$

[18] Salimbahrami B and Lohmann B 2002 Krylov subspace methods in linear model order reduction: introduction and invariance properties Scientific Report University of Bremen

[19] MUMPS Design Handbook 2002 MEMSCAP, Inc.

[20] Huang Q A and Lee N K S 2002 Analysis design of polysilicon thermal flexure actuator J. Micromech. Microeng. 9 64-70

[21] Holman J P 1989 Heat Transfer (Singapore: McGraw-Hill)

[22] Manginell R 1997 Polycrystalline-silicon micro-bridge combustible gas sensor PhD Dissertation, University of New Mexico, Mexico

[23] Mastrangelo C H 1991 Thermal applications of micro-bridges PhD Dissertation, University of California, Berkeley, USA

[24] Butler J and Bright V 1998 Electrothermal and fabrication modeling of polysilicon thermal actuators ASME DSC Micro-Electro-Mech. Syst. 66 571-6 